\title{
Discrete-time robust control with an anticipative action for preview systems
}

\author{
Asma Achnib ${ }^{1,2}$ \\ Tudor-Bogdan Airimitoaie* \\ Patrick Lanusse \\ Sergey Abrashov \\ ${ }^{1}$ Univ. Bordeaux, CNRS, IMS, UMR 5218 \\ Bordeaux INP, CNRS, IMS, UMR 5218 \\ 33405 Talence, France \\ Email: asma.achnib@u-bordeaux.fr \\ tudor-bogdan.airimitoaie@u-bordeaux.fr \\ patrick.lanusse@bordeaux-inp.fr \\ sergey.abrashov@u-bordeaux.fr \\ Mohamed Aoun \\ Manel Chetoui \\ 2 University of Gabes, National Engineering School of Gabes \\ Laboratoire Modélisation, Analyse et Commande des Systèmes - LR16ES22 \\ Email: mohamed.aoun@gmail.com \\ chetoui.manel@gmail.com
}

A discrete-time robust controller design method is proposed for optimal tracking of future references in preview systems. In the context of preview systems, it is supposed that future values of the reference signal are available a number of time steps ahead. The objective is to design a control algorithm that minimizes a quadratic error between the reference and the output of the system and at the same time achieves a good level of the control signal. The proposed solution combines a robust feedback controller with a feedforward anticipative filter. The feedback controller's purpose is to assure robustness of the closed-loop system to model uncertainties. Any robust control methodology can be used (such as $\mu$-synthesis, QFT, or CRONE control). The focus of this paper will be on the design of the feedforward action in order to introduce the anticipative effect with respect to known future values of the reference signal without hindering the robustness achieved through the feedback controller. As such, the model uncertainties are taken into account also in the design of the feedforward anticipative filter. The proposed solution is validated in simulation and on an experimental water tank level control system.

Keywords: preview systems, robust control, feedforward filter, discrete-time systems, $\mathcal{H}_{2}, \mathcal{H}_{\infty}$

${ }^{*}$ Corresponding author.

\section{Introduction}

In a number of automatic control applications (known also as preview systems), future values of the reference signal are available in advance. Examples can be found in aeronautical systems (trajectory tracking of unmaned aerial vehicles in [1]), autonomous vehicles (explicit path tracking in [2]) or mobile robots [3]. In other applications, disturbance signals can also be known beforehand, such as in water level control (for irrigation and drainage ducts in $[4,5]$ ). Therefore, it is of interest to propose appropriate control algorithms that take into account this information.

The present paper is focused on the development of discrete-time robust control methods with an anticipative action. Throughout this paper, it is considered that future values of the reference signal are known a number of time steps ahead. The objective of anticipation is that of designing control algorithms capable of minimizing a quadratic criterion on the error between the plant output and the known future reference. As such, the effect of anticipation is that the control input anticipates the future values of the reference signal so that the plant output reacts to reference changes before they occur.

The concept of anticipative control (known also as preview control) was proposed initially in [6] and further developed in [7]. A complete review of anticipative control is presented in [8]. Recent research has focused on the design of anticipative controllers in a $\mathcal{H}_{2}$ or $\mathcal{H}_{\infty}$ framework ( [9-14]). 
In [15], analytic solutions of operator Ricatti equations for preview/delay MIMO systems in an optimal control context are proposed. The focus is on the attenuation of disturbances given noisy measurement signals. Other control techniques have been used in combination with anticipative action such as multi-model adaptive control [16], fuzzy control [17] and sliding mode control [18].

Nevertheless, only a few number of research articles have attempted to deal with the problem of robust anticipative control. In [19] an anticipative control problem is considered in the context of motion control of robots. The proposed solution is also based on the $\mathcal{H}_{\infty}$ design methodology. Weighting norms are used to assure robustness of the feedback control law; however, uncertainties are not taken into account in the design of the feedforward anticipative filter. Furthermore, the anticipative feedforward part is designed taking into account only the tracking error minimization.

A free-weighting matrices technique is used with Lyapunov stability theory to derive robust anticipative controllers in $[8,20]$. Both reference anticipative tracking and disturbance rejection are treated; however, an initial design step involves a quadratic criterion which depends on weighting matrices that can be chosen arbitrarily. The proposed method is dependant on the choice of these weighting matrices, which makes it difficult to interpret the obtained results.

In this paper, a new robust anticipative controller design is proposed for preview systems. The robustness considerations take into account both parametric uncertainties and control effort limitations.

A two degrees of freedom feedforward-feedback control schema represents the basis for the proposed controller. A feedforward filter with anticipative behaviour is introduced to take into account future values of the reference signal. It is supposed that the time window of known future reference values is known. The preview window's length of the anticipative feedforward filter can be adjusted accordingly. The feedforward part is obtained by solving a frequency domain optimisation problem with mixed $\mathcal{H}_{2}$ performance and $\mathcal{H}_{\infty}$ constraints for robustness. The interesting aspect of the proposed approach is that the anticipative feedforward action can be added on top of any robust feedback controller. The presentation will be mainly focused on the feedforward part. With respect to the feedback part, in this paper a CRONE (french acronym for Commande Robuste d'Ordre Non Entier, which translates to Fractional Order Robust Control) robust controller is designed but it should be noted that any robust design methodology (such as $\mu$-synthesis, QFT, etc.) can be used instead. The CRONE methodology uses fractional orders of integro-differentiator to make easier the design of the robust controller. It does not require any overestimation of the plant uncertainty model, so it is able to design a high performing controller.

The paper is organised as follows. Notations used throughout the paper are introduced in Section 2. The basic principles of the proposed approach are introduced in Section 3 and then further developed in Section 3.3. Section 4 briefly recalls the design steps of a robust feedback CRONE controller and evaluates the proposed anticipative

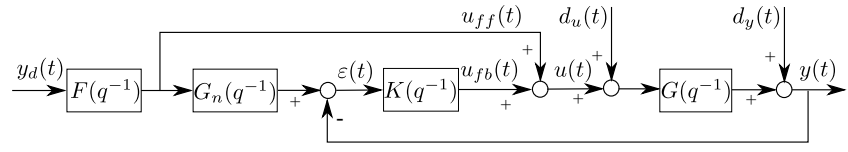

Fig. 1. Feedforward-feedback control schema used for robust anticipative control.

control method on an uncertain simulated system. A description of the experimental test bench used for validation and a discussion of the experimental results obtained are presented in Section 5. Section 6 concludes this paper.

\section{Notations}

In this paper, signals are denoted with lower-case letters in the time domain and upper-case letters in the frequency domain. Transfer functions or polynomials are also denoted with upper-case letters. The unit delay operator $q^{-1}$ is used in the time domain, while $z^{-1}$ is used in the frequency domain. The unit advance operator $q$ is used in the feedforward filters to represent the anticipative action. The sampling period is denoted by $T_{s}$, while the sampling frequency is represented by $f_{s}$.

As mentioned in the introduction, this paper deals with the design of robust anticipative control algorithms for reference tracking in preview systems. A feedforward-feedback discrete-time controller structure (see Fig. 1) represents the basis of the proposed control law.

In Fig. 1, $G\left(q^{-1}\right)$ represents the true plant model with uncertain parameters. It is supposed (without loss of generality) that the uncertainty intervals of the parameters are known. The true plant model can be considered as belonging to a set of models defined using the uncertainty intervals. The objective of robustness can be redefined as robustness to all the models in the model-set.

$G_{n}\left(q^{-1}\right)$ denotes a nominal model chosen from the model-set. The following factorization is considered for the nominal model

$$
G_{n}\left(q^{-1}\right)=G_{h}\left(q^{-1}\right) G_{l}\left(q^{-1}\right)
$$

where $G_{l}$ includes the low frequency stable poles and minimum phase zeros of $G_{n}$. All the other poles and zeros of $G_{n}$ are included in $G_{h}$. The difference between low and high frequency parts is based on the bandwidth of the system.

$G_{n}\left(q^{-1}\right)$ functions as a pre-filter in Fig. 1 (if $G_{n}\left(q^{-1}\right) \equiv$ $G\left(q^{-1}\right)$ and $d_{y}(t)=d_{u}(t)=0$ then $\varepsilon(t)=0$ and $u(t)=$ $\left.u_{f f}(t)\right)$.

$K\left(q^{-1}\right)$ is the robust feedback controller. $y_{d}(t), u_{f f}(t)$, $u_{f b}(t), u(t), y(t)$, and $d(t)$ represent, respectively, the reference input, the feedforward control, the feedback control, the control input, the system output, and the disturbance signal.

In this paper, the anticipative action is introduced using anticipative finite impulse response (FIR) filters defined as

$$
X\left(q^{-1}\right)=q^{-n_{X}} x_{-n_{X}}+\ldots+x_{0}+q^{1} x_{1}+\ldots+q^{m_{X}} x_{m_{X}} .
$$


In the previous equation, $m_{X} \cdot T_{s}$ determines the window of future time samples. FIR filters are preferred due to their inherent stability and ease of manipulation.

The anticipative action of the proposed controller with respect to known future values of the reference $y_{d}(t)$ is introduced through the anticipative FIR filter $F\left(q^{-1}\right)$ in Fig. 1.

Two closed-loop transfer functions in Fig. 1 are of interest. Let us define the transfer function from reference to system output, in the frequency-domain, as

$$
\frac{Y\left(z^{-1}\right)}{Y_{d}\left(z^{-1}\right)}=H_{y y_{d}}\left(z^{-1}\right)=G\left(z^{-1}\right) F\left(z^{-1}\right) \frac{1+K\left(z^{-1}\right) G_{n}\left(z^{-1}\right)}{1+K\left(z^{-1}\right) G\left(z^{-1}\right)}
$$

and the transfer function from reference to control input, also in the frequency-domain, as

$$
\frac{U\left(z^{-1}\right)}{Y_{d}\left(z^{-1}\right)}=H_{u y_{d}}\left(z^{-1}\right)=F\left(z^{-1}\right) \frac{1+K\left(z^{-1}\right) G_{n}\left(z^{-1}\right)}{1+K\left(z^{-1}\right) G\left(z^{-1}\right)},
$$

where $Y\left(z^{-1}\right), Y_{d}\left(z^{-1}\right)$ and $U\left(z^{-1}\right)$ are the Z-transforms of $y(t), y_{d}(t)$ and $u(t)$.

\section{Proposed anticipative controller using feedforward action}

In this section, the proposed design of the anticipative feedforward filter for preview systems is presented. The method is first introduced considering the nominal model $G_{n}$ and also the true plant model $G$ as known. In practice, the true plant is not known. Subsection 3.2 shows how the model-set described in Section 2 can be used to substitute the true plant model in the optimisation problem while assuring also the robustness of the obtained feedforward anticipative filter to model uncertainties. Finally, a method for reducing the number of parameters of the anticipative feedforward filter is explained in Subsection 3.3.

\subsection{Design of the anticipative feedforward filter}

Feedforward filter designs are usually based on some type of inversion of a plant model; however, in real applications, plant models are uncertain and present also noninvertible parts. In Section 2, the factorisation of the nominal model $G_{n}$ into the invertible $G_{l}$ and non-invertible $G_{h}$ parts has been introduced. To easily introduce the invertible part $G_{l}$ and also an anticipative action, the feedforward filter $F\left(q^{-1}\right)$ is factorised as:

$$
F\left(q^{-1}\right)=T_{F}\left(q^{-1}\right) F_{0}\left(q^{-1}\right)
$$

The $F_{0}$ factor in $F$ is used to compensate the low frequency stable poles and minimum phase zeros of $G_{n}$. It is computed as: $F_{0}\left(q^{-1}\right)=G_{l}^{-1}\left(q^{-1}\right)$. From (3), it can be seen that if $|K G| \gg 1$ and $\left|K G_{n}\right| \gg 1$ then $H_{y y_{d}}$ tends to $F G$, which motivates the choice for $F_{0} . T_{F}\left(q^{-1}\right)$ is chosen as anticipative
FIR filter:

$$
\begin{aligned}
T_{F}\left(q^{-1}\right)=t_{F_{-m}} q^{-m}+\ldots+t_{F_{-1}} q^{-1}+t_{F_{0}} & \\
& +t_{F_{1}} q+\ldots+t_{F_{a}} q^{a} .
\end{aligned}
$$

Due to $T_{F}\left(q^{-1}\right)$, both past and future data are used when filtering a signal through $F\left(q^{-1}\right) . m$ represents the number of data that have to be saved in the memory of the filter. $a$ gives the anticipation. A larger $a$ means that information further in the future is needed. While the choice of $m$ is limited only by the available memory, $a$ has to be adjusted taking into account the time window of available future reference values. In practice, $a$ and $m$ have to be adjusted in accordance to the desired dynamics of the controlled system and the desired closed-loop response time.

Let denote

$$
\theta_{F}=\left[t_{F_{-m}}, \ldots, t_{F_{-1}}, t_{F_{0}}, t_{F_{1}}, \ldots, t_{F_{a}}\right]
$$

the parameters vector of the unknown filter $T_{F}\left(q^{-1}\right)$. In the rest of this section, the problem of finding the optimal estimation $\hat{\theta}_{F}$ is considered. The notations $T_{F}\left(\hat{\theta}_{F}, q^{-1}\right)$ and $F\left(\hat{\theta}_{F}, q^{-1}\right)$ correspond to $T_{F}\left(q^{-1}\right)$ and $F\left(q^{-1}\right)$ when $\hat{\theta}_{F}$ is used.

The previously defined closed-loop transfer functions (3) and (4) can be rewritten as:

$$
\begin{aligned}
\frac{Y\left(z^{-1}\right)}{Y_{d}\left(z^{-1}\right)} & =H_{y y_{d}}\left(\hat{\theta}_{F}, z^{-1}\right) \\
& =G\left(z^{-1}\right) F\left(\hat{\theta}_{F}, z^{-1}\right) \frac{1+K\left(z^{-1}\right) G_{n}\left(z^{-1}\right)}{1+K\left(z^{-1}\right) G\left(z^{-1}\right)} \\
\frac{U\left(z^{-1}\right)}{Y_{d}\left(z^{-1}\right)} & =H_{u y_{d}}\left(\hat{\theta}_{F}, z^{-1}\right) \\
& =F\left(\hat{\theta}_{F}, z^{-1}\right) \frac{1+K\left(z^{-1}\right) G_{n}\left(z^{-1}\right)}{1+K\left(z^{-1}\right) G\left(z^{-1}\right)}
\end{aligned}
$$

Let $\varepsilon_{y}(t)=y_{d}(t)-y(t)=\left(1-H_{y y_{d}}\right) y_{d}(t)$ define the error between the system output and the reference signal. In order to minimize the $\infty-$ norm of $\varepsilon_{y}(t)$, the following objective function is defined

$$
\begin{aligned}
J_{F}\left(\hat{\theta}_{F}\right) & =\sup _{t}\left|\varepsilon_{y}(t)\right|=\sup _{t}\left|\varepsilon_{y}(t)\right| \\
& =\sup _{t}\left|\left(1-H_{y y_{d}}\left(\hat{\theta}_{F}\right)\right) y_{d}(t)\right|
\end{aligned}
$$

For finite energy reference signals $y_{d}(t)$, a least upper bound of the previous objective functions can be obtained by using the $\mathcal{H}_{2}$ system norm ${ }^{1}$ (see [21]).

$$
J_{F}\left(\hat{\theta}_{F}\right) \leq\left\|1-H_{y y_{d}}\left(\hat{\theta}_{F}, e^{-j 2 \pi f / f_{s}}\right)\right\|_{2}\left\|y_{d}(t)\right\|_{2}
$$

\footnotetext{
${ }^{1}$ In the frequency domain, the $q^{-1}$ operator becomes $e^{-j \omega T_{s}}=e^{-j 2 \pi f / f_{s}}$.
} 
Although it is supposed that a number of future samples of the reference signal are known at each time, the type and frequency content of the reference signal are supposed unknown. The minimization of $J_{F}\left(\hat{\theta}_{F}\right)$ is carried out for a given $y_{d}$. As such, we define the optimisation problem that needs to be solved using only the $\mathcal{H}_{2}$ system norm from (11) (which is equivalent with considering a normalization of $J_{F}\left(\hat{\theta}_{F}\right)$ by the norm $\left.\left\|y_{d}(t)\right\|_{2}\right)$ :

$$
\begin{aligned}
& \hat{\theta}_{F}=\arg \min _{\theta_{F}}\left\|1-H_{y y_{d}}\left(\theta_{F}, e^{-j 2 \pi f / f_{s}}\right)\right\|_{2}, \\
& \text { s.t. } \lim _{q \rightarrow 1} T_{F}\left(\hat{\theta}_{F}, q^{-1}\right)=\lim _{q \rightarrow 1} G_{h}^{-1}\left(q^{-1}\right) \\
& \text { and }\left\|W_{u y_{d}}\left(e^{-j 2 \pi f / f_{s}}\right) H_{u y_{d}}\left(\hat{\theta}_{F}, e^{-j 2 \pi f / f_{s}}\right)\right\|_{\infty} \leq 1 \\
& \quad \forall f \in\left[0, \frac{f_{s}}{2}\right]
\end{aligned}
$$

Two constraints are added to the optimisation problem in (12). The first one, $(12 \mathrm{~b})$, is introduced to ensure that the steady state gain of $F G_{n}$ has unit value.

In the second one, (12c), the weighting function $W_{u y_{d}}\left(q^{-1}\right)$ is used to introduce a frequency constraint on the control input $u_{f f}$. In practice, this is necessary in order to limit the control action at frequencies where the plant model has very low gain. In the example from Section 4, the control effort frequency constraint is used to reduce the frequency content of the control at high frequencies, where the plant model has very low gain.

\subsection{Robustness considerations in the design of the an- ticipative feedforward filter}

The closed-loop transfer functions $H_{y y_{d}}\left(\hat{\theta}_{F}, q^{-1}\right)$ and $H_{u y_{d}}\left(\hat{\theta}_{F}, q^{-1}\right)$ that appear in the optimisation problem (12) need the true plant model $G$ for their computation. As this is not known exactly, it should be replaced by the models from the model-set. As such, (8) and (9) become:

$$
\begin{array}{r}
H_{y y_{d}}\left(\hat{\theta}_{F}, q^{-1}\right)=\left\{G_{k} \frac{1+K G_{n}}{1+K G_{k}} F\left(\hat{\theta}_{F}\right),\right. \\
\left.\forall G_{k} \text { in the model-set }\right\}
\end{array}
$$

and

$$
\begin{aligned}
& H_{u y_{d}}\left(\hat{\theta}_{F}, q^{-1}\right)=\left\{\frac{1+K G_{n}}{1+K G_{k}} F\left(\hat{\theta}_{F}\right),\right. \\
& \left.\forall G_{k} \text { in the model-set }\right\} \text {. }
\end{aligned}
$$

(13) and (14) define two sets of transfer functions parametrized by the vector of parameters $\hat{\theta}_{F}$. The optimization problem (12) is redefined using (13) and (14) so that $\hat{\theta}_{F}$ should satisfy the criterion and the constraints for all $H_{y y_{d}}$ and $H_{u y_{d}}$ in the given sets.

Remark: in practice, it is possible to select only a smaller number of models from the model-set that contain the necessary information about the variations of the true plant for the design of the robust controller. The problem of how these models have to be selected is not dealt with in this paper. In Section 4, only the nominal model and two other models from the model-set are used.

\subsection{Reduction of the number of parameters of the an- ticipative feedforward filter}

In certain cases, the sampling period $T_{S}$ can be too small with respect to the desired closed-loop response of the system implying that $T_{F}\left(q^{-1}\right)$ would have too many parameters. It is possible to reduce its size by using a slower sampling period for $T_{F}$. Let $p$ denote a sampling time multiplier, then $T_{F}\left(q_{p}^{-1}\right)$ denotes the anticipative part of the feedforward filter that is sampled at $p \cdot T_{S}$ seconds:

$$
\begin{aligned}
T_{F}\left(q_{p}^{-1}\right)=t_{F_{-m}} q_{p}^{-m_{p}}+\ldots+t_{F_{-1}} q_{p}^{-1} & +t_{F_{0}}+t_{F_{1}} q_{p} \\
& +\ldots+t_{F_{a}} q_{p}^{a_{p}}
\end{aligned}
$$

In the previous equation, $q_{p}^{-1}$ defines a new delay operator that introduces a time shift of $p \cdot T_{s}$ seconds. It can be observed that using (15) a longer time span can be reached with fewer parameters. Taking into account the sampling period multiplier $p$, the sampling frequency for the $T_{F}\left(q_{p}^{-1}\right)$ filter in (15) is $\frac{f_{s}}{p}$. As such, $q_{p}^{-1}$ becomes in the frequency domain $e^{-j 2 \pi p f / f_{s}}\left(f \in\left[0, \frac{f_{s}}{2 p}\right]\right)$.

The optimisation problem in (12) involves filters $H_{y y_{d}}$ and $H_{u y_{d}}$. For $p \neq 1$, these transfer functions are not well defined in the time-domain as they involve both $q^{-1}$ and $q_{p}^{-1}$ delay operators. Nevertheless, in the frequency domain one can compute both the frequency response of the modified $T_{F}\left(\hat{\theta}, q_{p}^{-1}\right)$ filter and that of the filters in $q^{-1}$ that appear in (8) and (9) :

$$
\begin{aligned}
& H_{y y_{d}}^{\prime}\left(q^{-1}\right)=G\left(q^{-1}\right) \frac{1+K\left(q^{-1}\right) G_{n}\left(q^{-1}\right)}{1+K\left(q^{-1}\right) G\left(q^{-1}\right)} \\
& H_{u y_{d}}^{\prime}\left(q^{-1}\right)=\frac{1+K\left(q^{-1}\right) G_{n}\left(q^{-1}\right)}{1+K\left(q^{-1}\right) G\left(q^{-1}\right)}
\end{aligned}
$$

for $\forall f \in\left[0, \frac{f_{s}}{2 p}\right]$. The optimisation problem becomes:

$$
\begin{aligned}
& \hat{\theta}_{F}=\arg \min _{\theta_{F}}\left\|1-H_{y y_{d}}^{\prime}\left(e^{-j 2 \pi f / f_{s}}\right) T_{F}\left(\theta_{F}, e^{-j 2 \pi p f / f_{s}}\right)\right\|_{2}, \\
& \text { s.t. } \lim _{q_{p} \rightarrow 1} T_{F}\left(\hat{\theta}_{F}, q_{p}^{-1}\right)=\lim _{q \rightarrow 1} G_{h}^{-1}\left(q^{-1}\right) \\
& \left\|W_{u y_{d}}\left(e^{-j 2 \pi f / f_{s}}\right) H_{u y_{d}}^{\prime}\left(e^{-j 2 \pi f / f_{s}}\right) T_{F}\left(\hat{\theta}_{F}, e^{-j 2 \pi p f / f_{s}}\right)\right\|_{\infty} \leq 1
\end{aligned}
$$

$$
\forall f \in\left[0, \frac{f_{s}}{2 p}\right]
$$


The schema for the feedforward-feedback controller with sampling time multiplier $p$ is shown in Fig. 2. As it can be observed, the sampling period multiplication $p$ is applied only to the anticipative feedforward filter $T_{F}\left(q_{p}^{-1}\right)$. Both $G_{h}$ and $G_{l}^{-1}$ are implemented using $T_{s}$ as sampling period which allows to reduce the variations of $\varepsilon(t)$ and therefore limit the noise on the control.

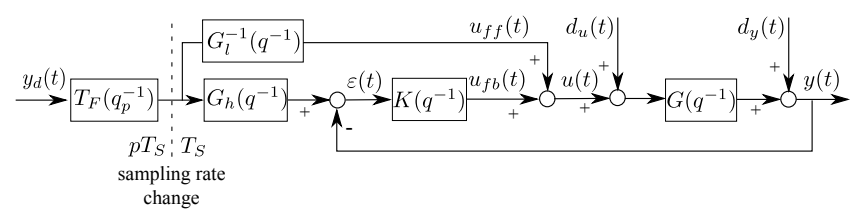

Fig. 2. Sampling rate change in the implementation of the feedforward-feedback controller. effects

Low-pass filter for attenuation of periodic control signal

The introduction of a sampling rate multiplier $p>1$ has the effect of limiting the frequency band which is used for the optimization problem to the interval $\left[0, \frac{f_{s}}{2 p}\right] \mathrm{Hz}$. To eliminate the undesired effects of aliasing from the $T_{F}\left(q_{p}^{-1}\right)$ above $\frac{f_{s}}{2 p}$, a low-pass interpolating filter $H_{f}\left(q^{-1}\right)$ with cut-off frequency $\frac{f_{s}}{2 p}$ is introduced with $T_{s}$ sampling period as shown in Fig. 3.

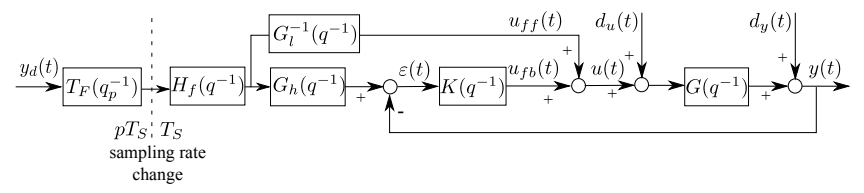

Fig. 3. Feedforward-feedback control schema used for robust anticipative control with sampling rate change and low-pass filter.

The optimization problem (12) is modified to take into account $H_{f}\left(q^{-1}\right)$ (due to the fact that it introduces phase changes below $\frac{f_{s}}{2 p}$ ). Equations (16) and (17) are modified as

$$
\begin{aligned}
& H_{y y_{d}}^{\prime}\left(q^{-1}\right)=G\left(q^{-1}\right) H_{f}\left(q^{-1}\right) \frac{1+K\left(q^{-1}\right) G_{n}\left(q^{-1}\right)}{1+K\left(q^{-1}\right) G\left(q^{-1}\right)} \\
& H_{u y_{d}}^{\prime}\left(q^{-1}\right)=H_{f}\left(q^{-1}\right) \frac{1+K\left(q^{-1}\right) G_{n}\left(q^{-1}\right)}{1+K\left(q^{-1}\right) G\left(q^{-1}\right)}
\end{aligned}
$$

Remark: for robustness considerations, the model-set defined in Subsection 3.2 is used instead of $G\left(q^{-1}\right)$ to solve the optimisation problem.

\section{Simulation results}

Simulation results on an academic example are presented in this section. First the uncertain parameters model

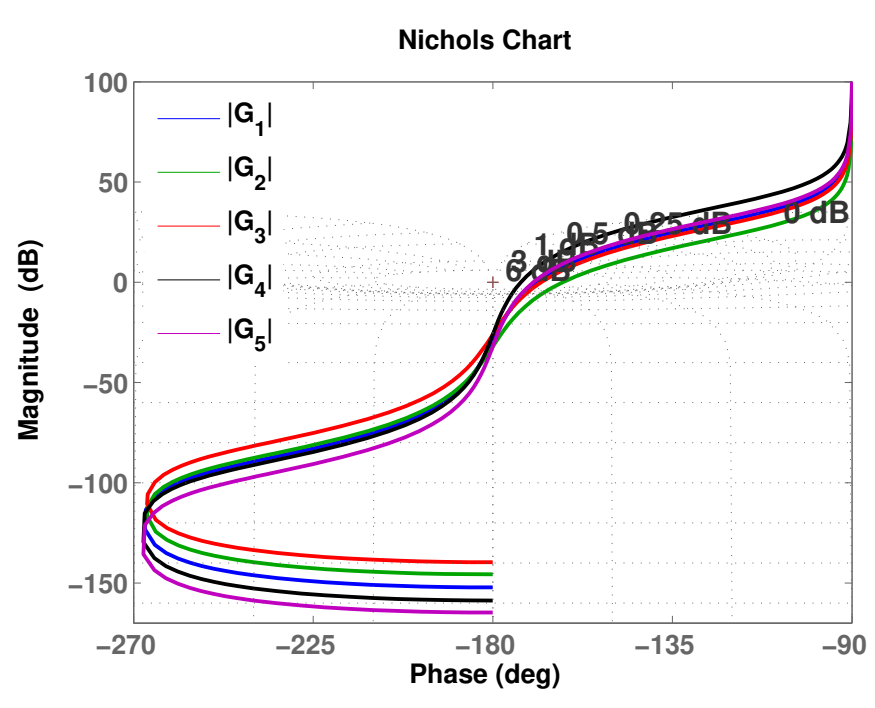

Fig. 4. Nichols chart of the uncertain parameters model $G\left(q^{-1}\right)$.

that will be used is introduced. Then the design of a robust feedback controller for the academic example based on the CRONE methodology is detailed. Finally the obtained anticipative feedforward-feedback controllers is evaluated.

\subsection{Uncertain parameters model example}

The discrete time plant model $G\left(q^{-1}\right)$ used for the design of the controllers and for simulations is obtained from the continuous-time model using a zero-order hold $(\mathrm{ZOH})$ discretization with $T_{s}=0.05 \mathrm{sec}$ of

$$
G(s)=\frac{k}{s(1+\tau s)}
$$

where $k \in[1,2], \tau \in[10,30]$ (sec) are the uncertain parameters.

The Nichols chart of the uncertain model $G\left(q^{-1}\right)$ is given in Fig. 4.

Generally, the choice of the nominal model from the model set is arbitrary. In this paper, the simulation and experimental examples use as nominal model the one that is the closest to the geometric mean of the frequency gain responses of the entire set. For this example, the nominal model is obtained by discretisation for $k=\sqrt{1 \cdot 2}$ and $\tau=\sqrt{10 \cdot 30}$ (sec)

$$
G_{n}\left(q^{-1}\right)=\frac{1.02 \cdot 10^{-4} q^{-1}+1.019 \cdot 10^{-4} q^{-2}}{1-1.997 q^{-1}+0.9971 q^{-2}}
$$

The two extreme models $G_{m}$ and $G_{M}$, for this example, are:

$$
\begin{aligned}
G_{m}\left(q^{-1}\right) & =\frac{4.164 \cdot 10^{-5}+4.4162 \cdot 10^{-5} q^{-1}}{1-1.998 q^{-1}+0.9983 q^{-2}} \\
G_{M}\left(q^{-1}\right) & =\frac{2.496 \cdot 10^{-4}+2.492 \cdot 10^{-4} q^{-1}}{1-1.995 q^{-1}+0.995 q^{-2}}
\end{aligned}
$$


4.2 Design of the robust feedback controller using CRONE methodology

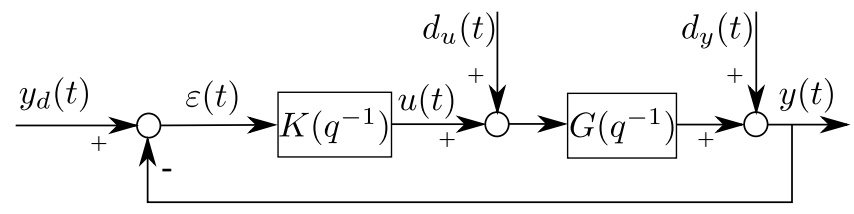

Fig. 5. Feedback schema used in CRONE controller design.

The objective of the CRONE methodology is to robustify the closed-loop dynamic performance through a robust stability degree (robust resonant peak) and performance. The theoretical background for designing a third generation CRONE controller [22] for the given uncertain parameters plant is presented in this section; however, the computation of the controller's parameters is done using the CRONE Toolbox [23].

Let us define the following sensitivity functions based on the schema in Fig. 5:

- Output sensitivity function

$$
\frac{Y\left(z^{-1}\right)}{D_{y}\left(z^{-1}\right)}=S\left(z^{-1}\right)=\frac{1}{1+G\left(z^{-1}\right) K\left(z^{-1}\right)} .
$$

- Complementary sensitivity function

$$
\frac{Y\left(z^{-1}\right)}{Y_{d}\left(z^{-1}\right)}=T\left(z^{-1}\right)=\frac{G\left(z^{-1}\right) K\left(z^{-1}\right)}{1+G\left(z^{-1}\right) K\left(z^{-1}\right)} .
$$

In the previous equations, $G\left(q^{-1}\right)$ can be any model from the model-set. It is possible to define $S_{n}\left(q^{-1}\right)$ and $T_{n}\left(q^{-1}\right)$ if the nominal model $G_{n}\left(q^{-1}\right)$ is used instead. For all other closedloop sensitivity functions in Fig. 5, a composed notation is used (for example, $K S_{n}\left(q^{-1}\right)$ denotes $K\left(q^{-1}\right) S_{n}\left(q^{-1}\right)$ ).

An aspect of CRONE design is that either continuous or pseudo-continuous transfer functions are used. As the objective is to find a discrete robust controller, the $\mathrm{ZOH}$ discretized plant model-set will be transferred to the pseudo-continuous space using the $\mathrm{W}$ bilinear variable change.

$$
q^{-1}=\frac{1-w}{1+w} .
$$

At the end, the inverse variable change will be used to get the discrete-time controller. Let us introduce the nominal openloop transfer function

$$
\begin{gathered}
\beta_{n}(w)=G_{n}(w) K(w)=C_{0} \beta_{l}(w) \beta_{m}(w) \beta_{h}(w)(1-w), \\
\beta_{l}(w)=\left(\frac{v_{0}}{w}+1\right)^{n_{l}}, \quad \beta_{h}(w)=\frac{1}{\left(\frac{w}{v_{1}}+1\right)^{n_{h}}},
\end{gathered}
$$

$\beta_{m}(w)=\left(\frac{1+w / v_{1}}{1+w / v_{0}}\right)^{a_{0}}\left(\Re_{i}\left\{\left(\alpha_{0} \frac{1+\frac{w}{v_{1}}}{1+\frac{w}{v_{0}}}\right)^{i b_{q_{0}}}\right\}\right)^{-q_{0} \operatorname{sign}\left(b_{q_{0}}\right)}$

with

$$
\alpha_{0}=\sqrt{\frac{1+\left(\frac{v_{r}}{v_{0}}\right)^{2}}{1+\left(\frac{v_{r}}{v_{1}}\right)^{2}}} .
$$

$w$ and $v$ denote, respectively, the operational variable in the pseudo-continuous time domain and the pseudo frequency.

Similarly to the continuous-time domain, $w=j v . v$ is related to the frequency $\omega$ in continuous-time through $v=$ $\tan \left(\frac{\omega T_{s}}{2}\right)$.

- $(1-w)$ takes into account the right half plane zero of $G_{n}(w)$ in order to ensure the stability of the $K S(w)$ transfer function.

- $n_{l}=3$ to ensure accuracy specifications at low frequencies (reducing the magnitude of the transfer $G S(w)$ (however, a single integrator will be used for the rational controller).

- $n_{h}=2$ to limit the high frequency control effort (biproper transfer function $K S(w)$ ).

- $a_{0}$ and $b_{0}$ are the real and imaginary parts of the fractional order $a_{0}+i b_{0}$ of band-limited integrator. $v_{0}$ and $v_{1}$ are their corner frequencies. The imaginary order $b_{q_{0}}$ and the positive integer order $q_{0}$ are determined to ensure the same open-loop phase slope as the slope ensured by an initial imaginary order $b_{0}$ which may have to be limited for closed-loop stability reasons [24]. For large values of $b_{0}, b_{q_{0}}$ is very close to $b_{0} / q_{0}$.

- From given values of the other parameters, $a_{0}, b_{q_{0}}, q_{0}$, and $C_{0}$ are deduced in order to ensure a resonant frequency $v_{r}$ and a desired resonant peak $M_{r d}$ of $\left|T_{n}(j v)\right|$. $v_{r}$ and $M_{r d}$ are used to tune the bandwidth and the damping of the nominal closed-loop transfer function $T_{n}(w)$.

The parameters of $\beta_{n}$ are optimized together to stabilize the nominal closed-loop and to minimize the resonant peak variations of $T(w)$ at the time of the plant perturbation. The objective function to be minimized is defined by:

$$
J_{K}=\sup _{v, G}|T(j v)|-M_{r d} .
$$

Only 5 parameters of $\beta_{n}$ need to be optimized: the $v_{0}$ and $v_{1}$ corner frequencies, the resonant frequency $v_{r}$, the resonant peak $M_{r d}, Y_{r}=\left|\beta_{n}\left(j v_{r}\right)\right|$. Setting the $M_{r d}$ desired resonant peak for $\left|T_{n}(j v)\right|$ makes the Nichols plot of $\beta_{n}(j v)$ tangent to the $M_{r d}$ Nichols M-contour. The minimization of $J_{K}$ optimizes the tangency direction in order to place the perturbed open-loop frequency response $\beta(j v)$ away from the $(-\pi, 0 \mathrm{~dB})$ critical point.

For the closed-loop performance requirement, a nonlinear minimization (using Matlab fmincon function) of $J_{K}$ is 
carried out thanks to 5 sets of frequency-domain inequality constraints:

$$
\begin{aligned}
\inf _{G}|T(j v)| & \geq T_{l}(v), \quad \sup _{G}|T(j v)| \leq T_{u}(v), \\
\sup _{G}|S(j v)| & \leq S_{u}(v), \\
\sup _{G}|K S(j v)| & \leq K S_{u}(v), \\
\sup _{G}|G S(j v)| & \leq G S_{u}(v) .
\end{aligned}
$$

As the perturbations of $G$ are taken into account without any overestimation (a set of LTI models), a non-conservative (highly efficient) robust controller can be designed. This modelling implies that a non-linear optimization method must be used to find the optimal values of the high-level descriptive parameters of fractional open-loop transfer function $\beta_{n}(w)$.

- $T_{l}(v)$ is defined by $-1 \mathrm{~dB}$ up to $v=0.05$ for fast convergence and a good-enough bandwidth. Similarly, $T_{u}(v)$ is defined by $+1 \mathrm{~dB}$ up to $v=0.05$ for fast convergence. From $0.05, T_{u}$ is defined by $4.5 \mathrm{~dB}$ to avoid obtaining a very low stability degree.

- $S_{u}(v)$ has a $+20 \mathrm{~dB} / \mathrm{dec}$ slope at low frequency to desensitize the closed-loop system with respect to plant uncertainties and a value of $6 \mathrm{~dB}$ at high frequency to obtain a modulus margin greater than 0.5 .

- $K S_{u}(v)$ has a value of $86 \mathrm{~dB}$ (20000 gain) to limit the high frequency amplification of the measurement noise on the control input.

- $G S_{u}(v)$ is defined by a $-40 \mathrm{~dB}$ gain to ensure good rejection of input disturbances.

One obtains the following values for the optimal parameters: $v_{0}=0.00505, v_{1}=3, v_{r}=0.1372, M_{r d}=1.74 \mathrm{~dB}$ and $Y_{r}=5.23 \mathrm{~dB}$. The deduced parameters are: $a_{0}=1.240$, $b_{0}=0.325, b_{q_{0}}=0.325, q_{0}=1$, and $C_{0}=27.1628$. The value of the objective function $J_{K}=0.92101 \mathrm{~dB}$.

The constraints used for the sensitivity functions (see (32)) are given in Fig. 6 together with the obtained results. The maximum (minimum) response is obtained considering at each frequency the highest (lowest) value over the entire model-set.

The Nichols chart of the obtained rational open-loop is compared to the fractional one in Fig. 7. Note that only 1 integrator is arbitrary used for the rational controller $(2$ integrators in the rational open-loop with respect to 3 for the fractional one).

Once $\beta_{n}(w)$ has been optimized, the controller is obtained from

$$
K(w)=G_{n}^{-1}(w) \beta_{n}(w) .
$$

As $\beta_{n}(w)$ is a fractional transfer function, an integer controller is obtained by identifying the ideal frequency response
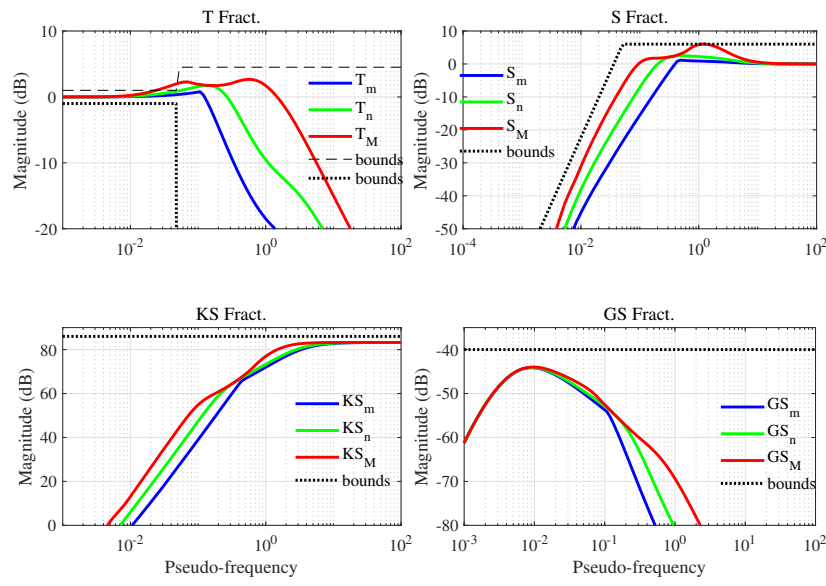

Fig. 6. Sensitivity functions templates and obtained results: subscripts denote the nominal response $(n)$, the highest response $(M)$, and the minimum response $(\mathrm{m})$
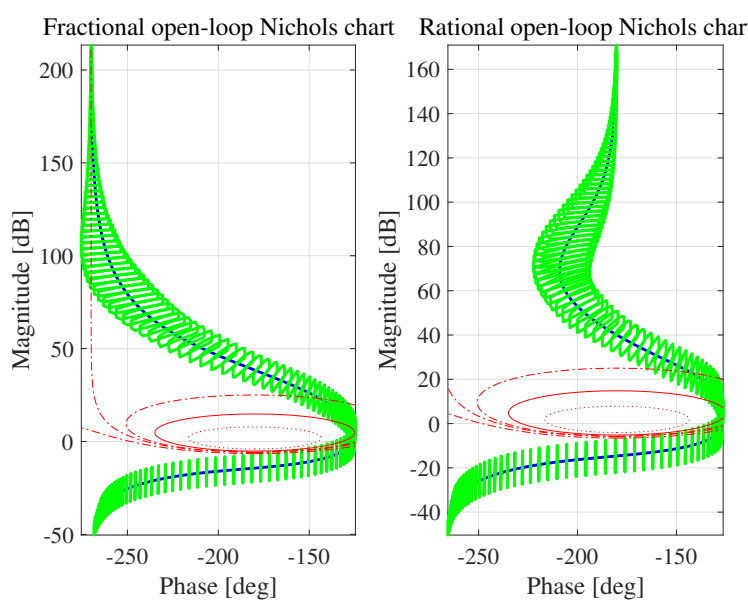

Fig. 7. Nichols chart comparison between the fractional and the rational open-loop: the nominal frequency responses are given by the solid lines. Responses of the augmented uncertainty domains enclose the nominal ones

$K(j v)$ by a low-order transfer function

$$
K_{R}(w)=\frac{K_{N}(w)}{K_{D}(w)},
$$

where $K_{N}(w)$ and $K_{D}(w)$ are polynomials of integer degrees $n_{K_{N}}$ and $n_{K_{D}}$. Whatever the complexity of the control problem, the method presented in [22, Chapter 3] (see also [23]) - optimization of the zeros and poles of a given rational transfer function - enables small enough values of $n_{K_{N}}$ and $n_{K_{D}}$ to be used. For this example, $n_{K_{N}}=n_{K_{D}}=3$. The discrete-time controller is obtained using the inverse of the bilinear variable change $w=\frac{1-q^{-1}}{1+q^{-1}}$. 
The rational discrete-time controller is obtained as

$$
K\left(q^{-1}\right)=\frac{3126-8314 q^{-1}+7297 q^{-2}-2108 q^{-3}}{1-1.223 q^{-1}-0.284 q^{-2}+0.5075 q^{-3}}
$$

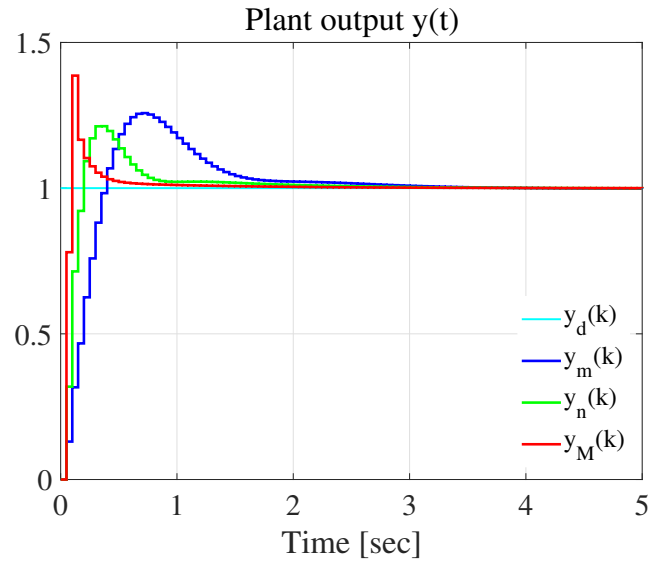

(a) Step response.

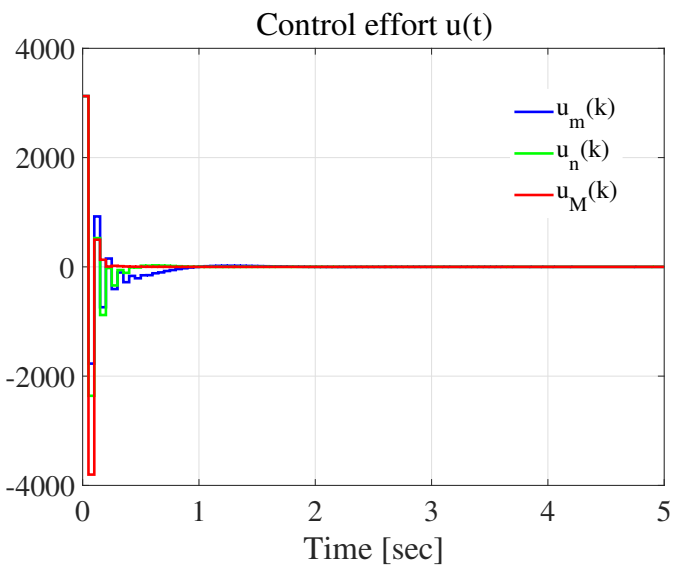

(b) Control effort.

Fig. 8. Evaluation of the rational CRONE controller.

Closed-loop time responses are compared in Fig. 8(a). The control input is shown Fig. 8(b). Note that, during this simulation no saturation effects have been considered and no noise/disturbance have been added.

From Fig. 8, it can be seen that the output tracks the reference signal for the three particular models of the plant and the control effort is an alternating signal with a higher amplitude. Also, the obtained results show that the synthesized controller is robust to the plant perturbation.

\subsection{Evaluation of the proposed anticipative approach}

The approach presented in Section 3 is validated on the same simulation example given in Subsection 4.1. The CRONE feedback controller presented in Subsection 4.2 is used.

The reference $y_{d}(t)$ is step function with unit amplitude.
The first results shown hereafter correspond to a 64 parameters $T_{F}\left(q^{-1}\right)$ filter with an anticipation order of $32(1.6$ $\mathrm{sec})$. The sampling time multiplier is chosen $p=1$. The expressions of $G_{h}$ and $G_{l}^{-1}$ are given, respectively, by

$$
\begin{aligned}
G_{h}\left(q^{-1}\right) & =0.000102 q^{-1}+0.0001019 q^{-2} \\
G_{l}^{-1}\left(q^{-1}\right) & =1-1.997 q^{-1}+0.9971 q^{-2}
\end{aligned}
$$

The weighting function $W_{u y_{d}}\left(q^{-1}\right)$ is a frequency constraint on the transfer from reference to control input. For an amplitude reference equal to 1 , we accept a control inputs level lower than 80. Then, the expression used in the optimisation problem is given in continuous-time by the following equation:

$$
W_{u y_{d}}^{-1}(s)=\frac{80}{\frac{s}{20}+1}
$$

The discretization is done using the Tustin transformation.

This results in a control input level limitation at 80 times the reference amplitude. The amplitude frequency response of this weighting function can be seen in Fig. 9. As mentioned in Subsection 3.1, $F_{0}\left(q^{-1}\right)=G_{l}^{-1}\left(q^{-1}\right)$. The coefficients of the $T_{F}\left(q^{-1}\right)$ filter are found by solving the linear optimization problem (12). This has been done by using the Yalmip toolbox for Matlab together with the MOSEK solver.

The anticipative FIR filter $T_{F}\left(q^{-1}\right)$ is obtained as

$$
\begin{aligned}
T_{F}\left(q^{-1}\right)=0.2308 q^{-31}+\ldots+202.6 q^{-1} \\
+227.6+246.8 q+\ldots+4.484 q^{32}
\end{aligned}
$$

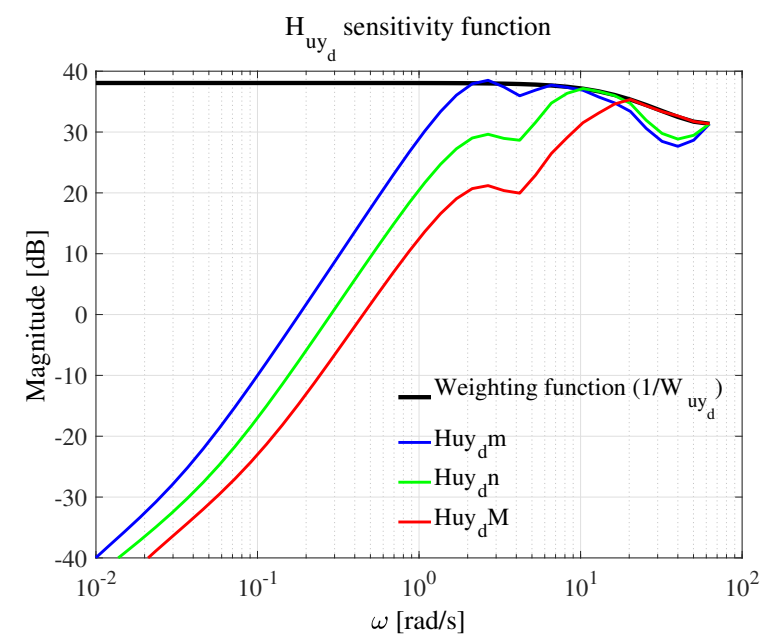

Fig. 9. Sensitivity functions from reference to control input using the nominal $G_{n}$ (labelled Huy $\mathrm{d}$ ) and the two extremum $G_{m}$ (labelled Huy $\left._{\mathrm{d}} \mathrm{m}\right)$ and $G_{M}$ (labelled Huy $\mathrm{d}$ M) plant models $(p=1)$

In all of the following figures, the subscript $n$ in the label represents the result obtained with the nominal model 
$G_{n}\left(q^{-1}\right)$. The subscripts $m$ and $M$ represent minimum (m) and maximum $(\mathrm{M})$ responses, respectively. From Fig. 9, it can be seen that the weighting constraint on the control signal is satisfied for reference tracking.

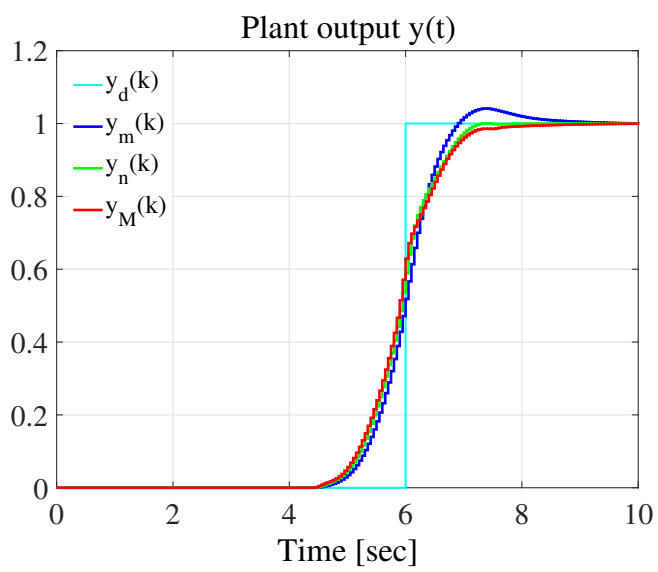

(a) Step response.

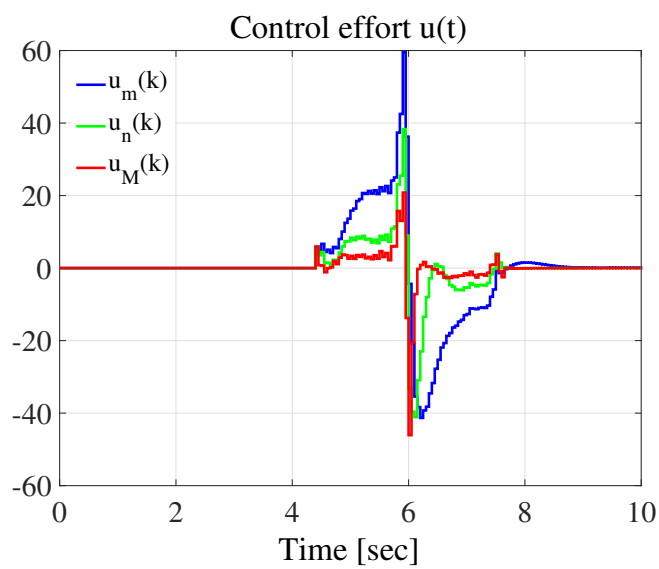

(b) Control effort.

Fig. 10. Evaluation of the feedforward + feedback control schema $(p=1)$.

From Fig. 9 it can be seen that the constraint on the control signal is satisfied. Step response and control input are shown in Figs. 10(a) and 10(b), respectively. The obtained results show that the anticipative effect with respect to known future values of the reference signal is validated. The control input is lower if compared to the control input obtained using only the feedback controller. The obtained control input level is, also, lower than 80 for different model-set which explains that the fixed constraint is respected. Furthermore, this approach is robust with respect to the uncertainty of the system.

The reduction of the number of coefficients is also tested. A sampling time multiplier $p=8$ is used here so that the number of coefficients of filter $T_{F}\left(q_{8}^{-1}\right)$ becomes 8 . A fourth-order low-pass interpolating filter with cut-off frequency equal to $1 \mathrm{~Hz}$ is used in order to avoid the important variations of $u_{f f}$ (see Section 3.3). The expression of this filter is given by the following equation

$$
H_{f}\left(q^{-1}\right)=\frac{10^{-4}\left(4+16 q^{-1}+25 q^{-2}+16 q^{-3}+4 q^{-4}\right)}{1-3.18 q^{-1}+3.86 q^{-2}-2.11 q^{-3}+0.44 q^{-4}}
$$

The anticipative filter $T_{F}\left(q_{8}^{-1}\right)$ is obtained as

$$
\begin{array}{r}
T_{F}\left(q_{8}^{-1}\right)=103.7 q_{8}^{-3}+140.7 q_{8}^{-2}+538.5 q_{8}^{-1}+615 \\
+1556 q_{8}+1236 q_{8}^{2}+479.1 q_{8}^{3}+236 q_{8}^{4}
\end{array}
$$

Remark that the unit delay operator's sampling period for $T_{F}\left(q_{8}^{-1}\right)$ is $p \cdot T_{s}$.

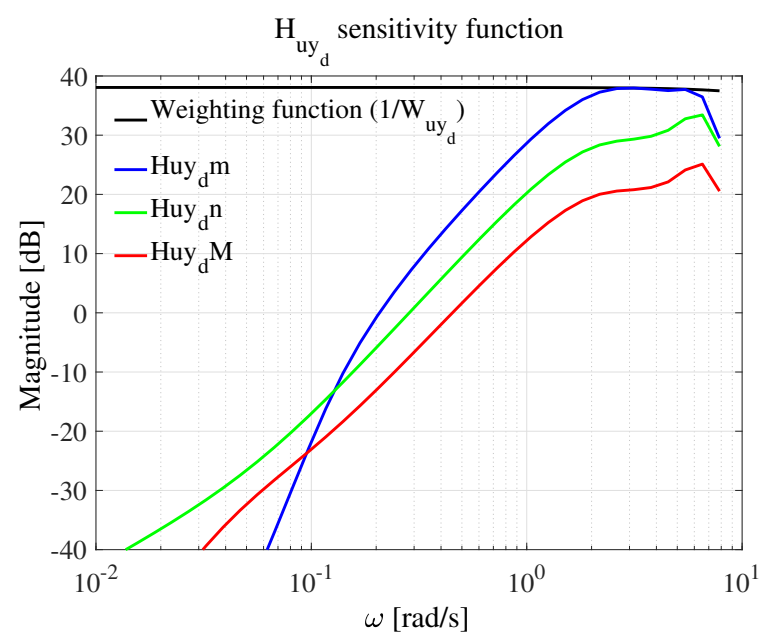

Fig. 11. Sensitivity functions from reference to control input using the nominal $G_{n}$ (labelled Huy $\mathrm{d}$ ) and the two extremum $G_{m}$ (labelled Huy $\left._{\mathrm{d}} \mathrm{m}\right)$ and $G_{M}$ (labelled Huy $\mathrm{d}$ M) plant models $(p=8)$

The control input sensitivity functions are shown in Fig. 11. The step response and the control input are given in Figs. 12(a) and 12(b), respectively.

The obtained results show that the constraint on the control signal is satisfied. Also, the output tracks well the reference and the control effort anticipates the reference variation. This control effort is, also, lower than 80 for different model-set. Furthermore, the robustness with respect to the uncertainty of the system the robustness is achieved.

\section{Experimental test bench}

In this section, we apply the proposed anticipative feedforward design methods on an experimental water tank level control test bench. This experimental test bench is described in Subsection 5.1. The obtained experimental results for the anticipative feedforward-feedback controllers using a CRONE feedback controller presented in [25] are discussed in Subsection 5.2. 


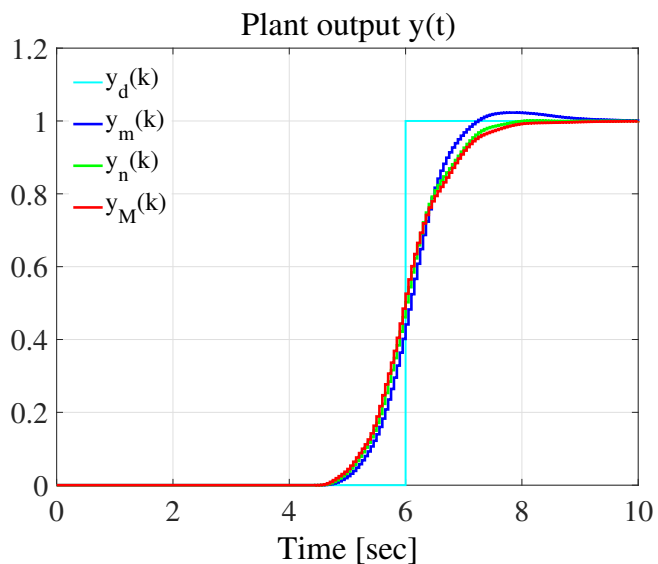

(a) Step response.

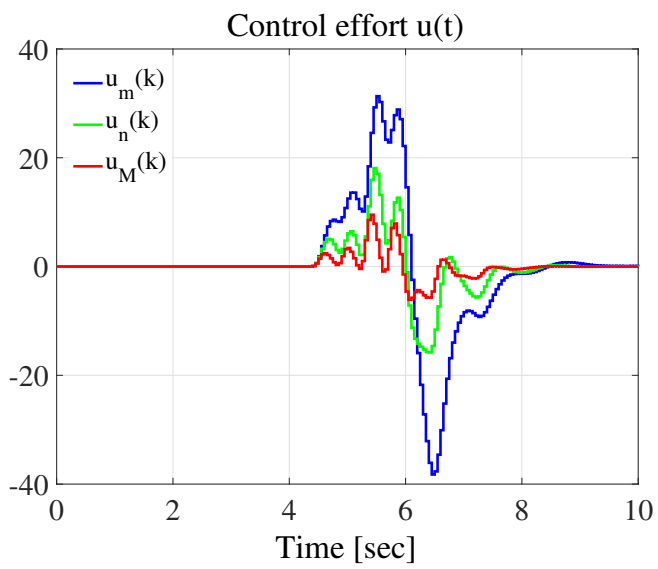

(b) Control effort.

Fig. 12. Evaluation of the feedforward + feedback control schema $(p=8)$.

\subsection{Description of the water tank test bench}

The water level control part of a Festo didactic test bench has been used for the experimental validation. A schematic representation is given in Fig. 13. Sensor S101 provides the measure of the output to be controller. Pump P101 is used to adjust the filling of tank T102. The valve

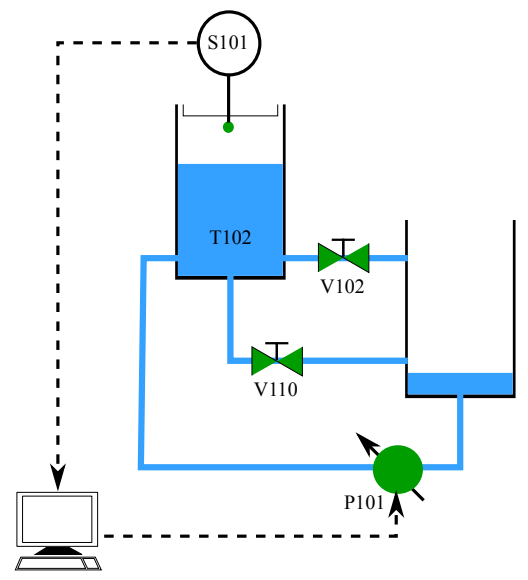

Fig. 13. Schema of the water level control test bench.
V110 is partially open throughout the experiments presented in this section.

The dynamic behavior of the water level in the tank is nonlinear as it depends on the water level. It is also uncertain as the valve V102 can be used in three positions: wide open, partially open and closed. For controller design, an identification of the test bench has been performed. A total of 18 first order continuous-time linear models have been obtained: 6 operating points for each one of the 3 positions of the V102 valve. Each operating point corresponds to a certain level of water in tank T102, going from 3 to 9 litres. The static gain and the time constant for the various first order plant models vary in the intervals: $k \in(0.118,1.7)(\mathrm{L} / \mathrm{V})$ and $\tau \in(19.61,205.8)$ (sec).

An average nominal model is defined:

$$
G_{n}(s)=\frac{0.332}{1+52.3169 s} .
$$

\subsection{Experimental results}

For this example, a third generation CRONE controller has been designed in [25] using the CRONE Toolbox [23]. Moreover, an anti-windup mechanism is added to the controller in order to deal with the problem of actuator saturation that persists. This anti-windup mechanism is based on an inner loop that feedbacks the integral part of the controller [26].

An anticipative feedforward filter with 80 parameters has been computed. The anticipation introduced corresponds to a duration of $40 \mathrm{sec}$ (order 40, sampling time $T_{s}=1 \mathrm{sec}$, $m=39$ and $a=40$ ). In all of the following figures, the subscript $n$ represents the result obtained with the nominal model $G_{n}$. The subscripts $m$ and $M$ denote the results for two other models from model set.

The discrete-time nominal model is obtained from (40) (z-transform and $\mathrm{ZOH}$ ):

$$
G_{n}\left(q^{-1}\right)=\frac{0.006286 q^{-1}}{1-0.9811 q^{-1}}
$$

The polynomials $G_{h}$ and $G_{l}^{-1}$ are given by

$$
\begin{gathered}
G_{h}\left(q^{-1}\right)=0.006286 q^{-1} \\
G_{l}^{-1}\left(q^{-1}\right)=1-0.9811 q^{-1}
\end{gathered}
$$

The anticipative FIR filters $T_{F}\left(q^{-1}\right)$ is obtained by solving the linear optimization problem (12)

$$
T_{F}\left(q^{-1}\right)=0.457 q^{-39}+\ldots+3.342+\ldots+0.4716 q^{40}
$$

As mentioned in Section 3.1, $F_{0}\left(q^{-1}\right)=G_{l}^{-1}\left(q^{-1}\right)$. A constraint of $6 \mathrm{~dB}$ defining $W_{u y_{d}}^{-1}$ has been imposed to the 


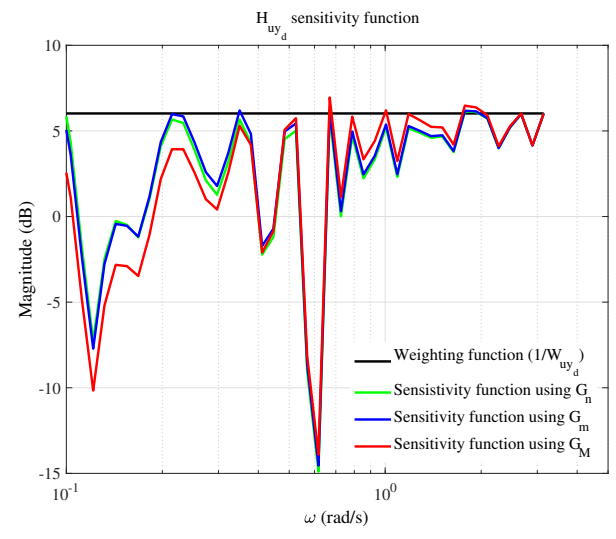

Fig. 14. Sensitivity functions from reference to control input using the nominal $G_{n}$ and the two extremum $G_{m}$ and $G_{M}$ plant models $(p=1)$

control input sensitivity function above $0.1 \mathrm{rad} / \mathrm{s}$. From Fig. 14 it can be seen that this constraint is globally satisfied for the three particular models of the plant.

First the V102 valve is considered wide open. Experimental results are shown in Fig.15. Two step changes in the reference signal are tested. The first one is from 2 to 2.5 litres and the second from 2.5 to 3 litres. Good tracking results can be observed even in the presence of control input saturation (see Fig. 15): anticipative and robust control of the volume.
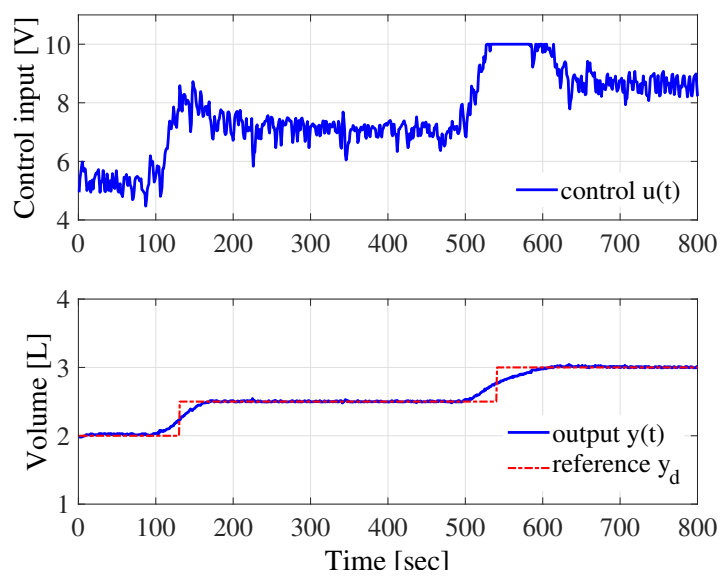

Fig. 15. Experimental results for the V102 manual valve wide open: control signal (upper plot), reference and output signals (lower plot).

In a second experiment, the V102 valve is considered partially open (Fig. 16). Thus it is possible to track greater water volume reference signals. As such, in this experiment the reference varies from 2 to 4.5 litres by steps of 0.5 litres. As can be observed in Fig. 16, the output tracks the reference signal efficiently.

Finally, the V102 manual valve is completely closed. This allows to fill the water tank up to 8 litres. As before, step changes of 0.5 litres tests have been done; however, only two of the step variations from 6 to 6.5 litres and from 6.5 to
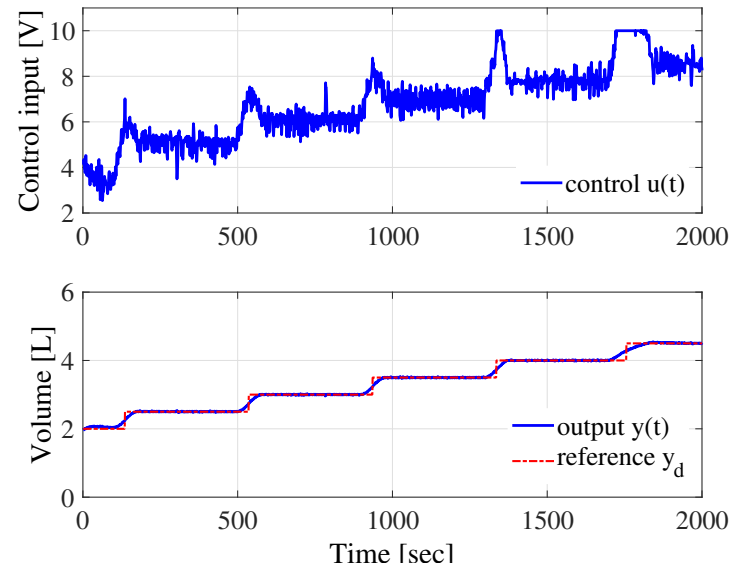

Fig. 16. Experimental results for the V102 manual valve partially open: control signal (upper plot), reference and output signals (lower plot).

7 litres are shown in Fig. 17.
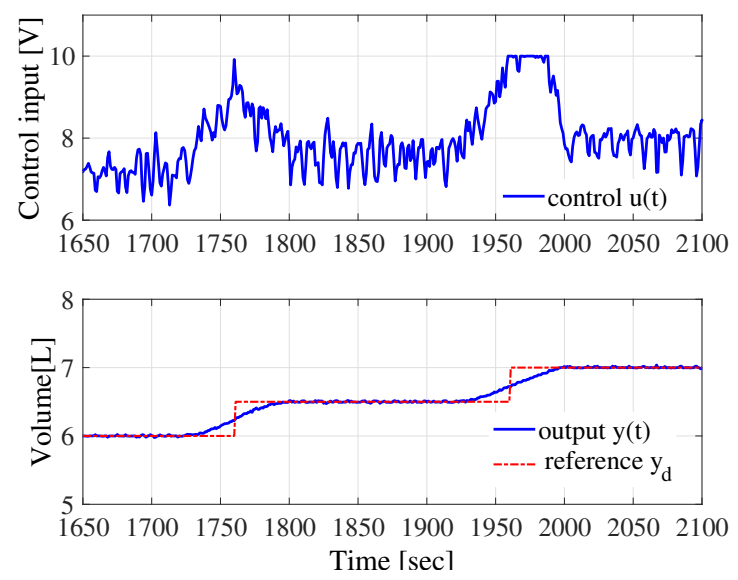

Fig. 17. Experimental results for the $\mathrm{V} 102$ manual valve completely closed: control signal (upper plot), reference and output signals (lower plot).

These experimental results show the robustness capabilities of the proposed approach. Despite significant changes in the plant frequency response for the various operation points and apertures of the V102 valve, and also the saturation of the control signal, the output of the system is efficiently tracking the desired reference trajectory.

Lastly, the anticipative effect of the feedforward filter is also noticeable in all experimental results. As expected, a $40 \mathrm{sec}$ anticipation in introduced.

\section{Conclusion}

In this paper, a controller design method for robust tracking of future reference signals in the context of preview systems has been presented. The proposed solution 
is based on a feedback-feedforward two degrees of freedom controller. The feedforward part uses an anticipative filter which is design in the frequency domain using a mix of $\mathcal{H}_{2}$ performance and $\mathcal{H}_{\infty}$ constraints to ensure tracking of future references. Furthermore, robustness with respect with uncertain parameters is considered in the design of the proposed controller. Then, the reduction of the number of parameters of the anticipative filter by using a slower sampling period for this anticipative FIR filter is treated. This new approach is validated on a simulated example and on an experimental water tank level control test bench. The obtained results validate the robustness of the proposed approach with respect to plant uncertainties.

In future works, this new approach will be extended to the case of rejection of future known disturbances and multiple-input multiple-output preview systems.

\section{Acknowledgements}

This work was supported by the Ministry of the Higher Education and Scientific Research in Tunisia.

\section{References}

[1] Park, S., Deyst, J., and How, J., 2004. "A new nonlinear guidance logic for trajectory tracking”. In AIAA Guidance, Navigation, and Control Conference and Exhibit, American Institute of Aeronautics and Astronautics.

[2] Shin, D. H., Singh, S., and Lee, J. J., 1992. "Explicit path tracking by autonomous vehicles". Robotica, 10, 11, pp. 539-554.

[3] Freitas, G., Lizarralde, F., Hsu, L., and Bergerman, M., 2013. "Terrain model-based anticipative control for articulated vehicles with low bandwidth actuators". In Robotics and Automation (ICRA), 2013 IEEE International Conference on, pp. 382-389.

[4] Schuurmans, J., Hof, A., Dijkstra, S., Bosgra, O. H., and Brouwer, R., 1999. "Simple water level controller for irrigation and drainage canals". Journal of Irrigation and Drainage Engineering, 125(4), pp. 189-195.

[5] Li, Y., 2014. "Offtake feedforward compensation for irrigation channels with distributed control". IEEE Transactions on Control Systems Technology, 22(5), Sept, pp. 1991-1998.

[6] Sheridan, T. B., 1966. "Three models of preview control". IEEE Transactions on Human Factors in Electronics(2), pp. 91-102.

[7] Tomizuka, M., 1975. "Optimal continuous finite preview problem”. IEEE transactions on automatic control, 20(3), pp. 362-365.

[8] Liao, F., and Li, L., 2016. "Robust preview tracking control for a class of uncertain discrete-time systems". Cogent Engineering, 3(1), p. 1243033.

[9] Kojima, A., and Ishijima, S., 2003. " $H_{\infty}$ performance of preview control systems". Automatica, 39(4), pp. 693701.

[10] Middleton, R. H., Chen, J., and Freudenberg, J. S., 2004. "Tracking sensitivity and achievable $H_{\infty}$ per- formance in preview control". Automatica, 40(8), pp. 1297-1306.

[11] Kojima, A., 2004. " $H_{2}$ performance on preview feedforward action". In Proceedings of the 16th MTNS.

[12] Moelja, A. A., and Meinsma, G., 2006. "H2 control of preview systems". Automatica, 42(6), pp. 945-952.

[13] Akbari, A., and Lohmann, B., 2010. "Output feedback $\mathrm{H}_{\infty} / \mathrm{GH}_{2}$ preview control of active vehicle suspensions: a comparison study of lqg preview". Vehicle System Dynamics, 48(12), pp. 1475-1494.

[14] Moran Cardenas, A., Rázuri, J. G., Bonet, I., Rahmani, R., and Sundgren, D., 2014. "Design of high accuracy tracking systems with $H_{\infty}$ preview control". Polibits(50), pp. 21-28.

[15] Kojima, A., 2015. " $H_{\infty}$ controller design for preview and delayed systems". IEEE Transactions on Automatic Control, 60(2), pp. 404-419.

[16] Wang, D., Liao, F., and Tomizuka, M., 2016. “Adaptive preview control for piecewise discrete-time systems using multiple models". Applied Mathematical Modelling, 40(23), pp. 9932-9946.

[17] Cheng, J., Dong, X., Xue, J., Wang, X., Wang, X., and Zhi, J., 2014. "Fuzzy preview controller design for aircraft-pilot closed loop system". Acta Aeronautica et Astronautica Sinica, 35(3), pp. 807-820.

[18] Williams, M. M., Loukianov, A. G., and BayroCorrochano, E., 2013. "Zmp based pattern generation for biped walking using optimal preview integral sliding mode control". In Humanoid Robots (Humanoids), 2013 13th IEEE-RAS International Conference on, IEEE, pp. 100-105.

[19] Makarov, M., Grossard, M., Rodríguez-Ayerbe, P., and Dumur, D., 2016. "Modeling and preview $H_{\infty}$ control design for motion control of elastic-joint robots with uncertainties". IEEE Transactions on Industrial Electronics, 63(10), pp. 6429-6438.

[20] Li, L., Liao, F., and Deng, J., 2017. " $H_{\infty}$ preview control of a class of uncertain discrete-time systems". Asian Journal of Control.

[21] Doyle, J. C., Francis, B. A., and Tannenbaum, A. R., 1990. Feedback control theory. Macmillan Publishing Co.

[22] Sabatier, J., Lanusse, P., Melchior, P., and Oustaloup, A., 2015. Fractional Order Differentiation and Robust Control Design. Intelligent Systems, Control and $\mathrm{Au}-$ tomation: Science and Engineering. Springer Netherlands.

[23] Lanusse, P., 2010. CRONE Control System Design, a CRONE toolbox for Matlab.

[24] Lanusse, P., Oustaloup, A., and Pommier-Budinger, V., 2012. "Stability of closed loop fractional order systems and definition of damping contours for the design of controllers". International Journal of Bifurcation and Chaos, 22(04), p. 1230013.

[25] Achnib, A., Airimitoaie, T.-B., Lanusse, P., Guefrachi, A., Aoun, M., and Chetoui, M., 2018. "Anticipative robust design applied to a water level control system". In European Control Conference (ECC). 
[26] Lanusse, P., Sabatier, J., and Oustaloup, A., 2015. "fractional order pid and first generation crone control system design". In Fractional Order Differentiation and Robust Control Design. Springer, pp. 63-105. 